\title{
Embryonic Regulation of the Mouse Hematopoietic Niche
}

\author{
Daisuke Sugiyama, ${ }^{1}$ Tomoko Inoue-Yokoo,,${ }^{1,2}$ Stuart T. Fraser, ${ }^{3}$ Kasem Kulkeaw, ${ }^{1}$ \\ Chiyo Mizuochi, ${ }^{1}$ and Yuka Horio ${ }^{1}$ \\ ${ }^{1}$ Division of Hematopoietic Stem Cells, Advanced Medical Initiatives, \\ Department of Advanced Medical Initiatives, Faculty of Medical Sciences, \\ Kyushu University, Fukuoka 812-8582, Japan \\ ${ }^{2}$ Department of Medicine and Biosystemic Science, Graduate School of \\ Medical Sciences, Kyushu University, Fukuoka 812-8582, Japan \\ ${ }^{3}$ Discipline of Physiology, School of Medical Sciences, University of Sydney, \\ Camperdown, NSW 2050, Australia
}

Received 16 July 2011; Accepted 16 September 2011

Academic Editor: David Tannahill

\begin{abstract}
Hematopoietic stem cells (HSCs) can differentiate into several types of hematopoietic cells (HCs) (such as erythrocytes, megakaryocytes, lymphocytes, neutrophils, or macrophages) and also undergo self-renewal to sustain hematopoiesis throughout an organism's lifetime. HSCs are currently used clinically as transplantation therapy in regenerative medicine and are typically obtained from healthy donors or cord blood. However, problems remain in HSC transplantation, such as shortage of cells, donor risks, rejection, and graft-versus-host disease (GVHD). Thus, increased understanding of HSC regulation should enable us to improve HSC therapy and develop novel regenerative medicine techniques. HSC regulation is governed by two types of activity: intrinsic regulation, programmed primarily by cell autonomous gene expression, and extrinsic factors, which originate from so-called "niche cells" surrounding HSCs. Here, we focus on the latter and discuss HSC regulation with special emphasis on the role played by niche cells.
\end{abstract}

KEYWORDS: embryonic hematopoiesis, hematopoietic stem cell, niche 


\section{INTRODUCTION}

After gastrulation, three germ layers emerge: endoderm, mesoderm, and ectoderm. Among them, mesoderm is the ancestor of hematopoietic cells ( $\mathrm{HCs}$ ), and both extraembryonic and intraembryonic mesoderm contribute to $\mathrm{HC}$ generation. In the mouse, the site of blood cell production changes as embryos develop over an approximately 20-day gestation period (Figure 1(a)). Hematopoiesis begins in the yolk sac (YS), producing mainly primitive erythroid cells at 7.5 days postcoitum $(\mathrm{dpc})$. Shortly thereafter, definitive myeloerythroid progenitor cells appear in the YS (around $8.25 \mathrm{dpc}$ ), which then seed the fetal liver (FL). This process, termed primitive hematopoiesis, diminishes at $12.5 \mathrm{dpc}$, when definitive hematopoiesis, which sustains the adult blood system through HSCs, begins in the FL. Although there is controversy over where HSCs are generated-in the extraembryonic mesoderm at the YS or in the intraembryonic para-aorticsplanchnopleural mesoderm (P-Sp)/aorta-gonad-mesonephros (AGM) region—recent studies suggest that both regions contain HSCs capable of reconstituting adult bone marrow hematopoiesis (Figures 1(a) and 1(b)). In addition, HSCs are generated in the placenta (PL), likely independent of the YS and P-Sp/AGM regions. Thereafter, these HSCs are thought to circulate and colonize the FL, where HSC expansion occurs. HSCs then temporarily reside in fetal spleen and finally shift to fetal bone marrow (BM) [1] (Figures 1(a) and $1(b))$.

\section{THE YOLK SAC}

The YS is a bilaminar membranous sac encasing the embryo proper. It is here the "blood islands," the first site of hematopoiesis. The YS is composed of two distinct layers of cells, the visceral endoderm which is predominantly an absorptive epithelium transporting nutrients to the embryo and the extraembryonic mesoderm which gives rise to the endothelium of the YS and the blood cells that fill these early vessels [2]. Developing mesoderm cells can be programmed either by the visceral endoderm or by soluble factors produced by the visceral endoderm such as Indian hedgehog (Ihh) to become hematopoietic or vascular cells $[3,4]$ (Figure 2). The extraembryonic mesoderm gives rise to the first hematopoietic cells in the developing conceptus. These include the primitive erythroid cells, macrophages, and megakaryocytes and appear shortly after gastrulation [5]. The primitive erythroid (EryP) cells are larger than their adult-type definitive counterparts (EryD) and express a unique set of embryonic globin genes [6]. EryP dominate the mid-gestation circulation of the mouse embryo and number in the several millions; however, by birth, they will be greatly reduced in frequency, diluted by the vast numbers of EryD generated in the FL [7, 8].

The niche supporting early hematopoietic development in the YS is poorly defined. However, recently some players in this field have been identified. YS endothelial cells and the visceral endoderm express the soluble growth factor angiopoietin 1, whereas early EryP cells express the angiopoietin receptor Tie-2. Interestingly, the EryP cells themselves express soluble growth factors [9]. TGFb1 expression was detected in the visceral endoderm and the EryP, suggesting that the developing hematopoietic cells may be generating a unique niche themselves. The hypoxic nature of the developing YS is also supportive of hematopoiesis $[9,10]$. As the YS develops, a broad range of hematopoietic progenitors are generated. These include definitive erythroid progenitors, myeloid progenitors, and lymphoid progenitors $[5,11,12]$. The migration of hematopoietic progenitors throughout the developing embryo has been investigated capitalizing on knockout mouse strains lacking a heartbeat and subsequent blood flow. These mice lacked the sodium/calcium exchanger Ncx1, failed to develop a heartbeat, and subsequently hematopoietic progenitors remained lodged at their sites of origin, namely, the YS [13] and the placenta [14]. Whether the progenitors isolated in the Ncx-1 knockout placenta are truly HSC is yet to be resolved [14]. Shortly before mid-gestation in the mouse embryo, long-term reconstituting HSCs appear in the YS [15]. These cells show significant differences compared to the HSCs generated within the embryo; however, they are capable of giving rise to the hematopoietic system of newborn mice $[15,16]$. The origin of HSC has proven a contentious issue; however, the development of genetic models to monitor HSC sites of production has 

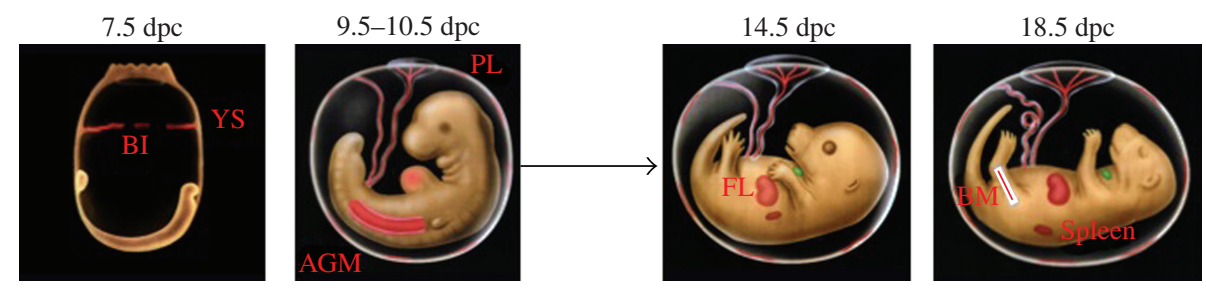

(a)

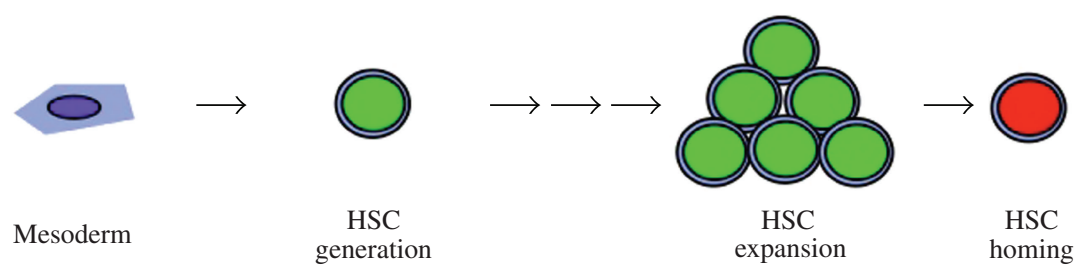

Modified from "Sugiyama D, Tsuji K et al. Trends in Cardiovascular Medicine, 2006"

(b)

FIGURE 1: Schema of hematopoietic development during mouse embryogenesis. (a) The site of blood cell production changes as embryos develop over an approximately 20-day gestation period in mice. Abbreviations: dpc (day post coitum), YS (yolk sac), BI (blood island), AGM (aorta-gonad-mesonephros), PL (placenta), FL (fetal liver), BM (bone marrow), HSC (hematopoietic stem cell). (b) Corresponding to the time course shown in (a), HSCs emerge in the YS, AGM region, and PL from early to mid-gestation (7.0$11.0 \mathrm{dpc}$ ). HSCs proliferate in the FL at mid-gestation and then home to fetal spleen temporarily and to fetal $\mathrm{BM}$ at late gestation.

supported the YS as, at least one, source of HSC during embryogenesis. Genetic "tagging and tracing" of early hematopoietic progenitors expressing the transcriptional regulator Runx1 in the YS suggests that this may indeed be a site of HSC production during embryogenesis [17].

\section{THE AGM REGION}

After YS hematopoiesis, HSC activity is detected in the p-Sp/AGM region, where cell aggregates, termed $\mathrm{HC}$ clusters or intra-aortic/arterial clusters (IACs), are observed. HSCs in the AGM region express cKit/CD117 (the receptor for stem cell factor), CD31/PECAM-1 (platelet/endothelial cell adhesion molecule 1), and CD34 (a transmembrane glycoprotein). Presumptive pre-HSCs expressing c-Kit and capable of reconstituting neonatal recipients are detected in the p-Sp/AGM region at $9.5 \mathrm{dpc}$, whereas functional cKit-expressing HSCs capable of reconstituting adult recipients are first identified in the AGM region at $10.5 \mathrm{dpc}$. Special microenvironments likely enable pre-HSCs from the p-Sp/AGM region at $9.5 \mathrm{dpc}$ to acquire functional HSC activity by $10.5 \mathrm{dpc}$. In the AGM region, IACs are frequently observed attached to floors and walls of large arteries, suggesting that they are generated from EC layers (Figure 3(a)). Mouse IACs have been characterized morphologically and are primarily located in the dorsal aorta, the omphalomesenteric artery, and the umbilical artery. Intrinsic regulation of AGM HSCs has been well characterized. The transmembrane receptor encoded by Notchl is essential to generate HSCs from ECs [18], and the ubiquitin ligase Mind bomb-1 (encoded by Mib1), which regulates Notch ligands, is important for intraembryonic hematopoiesis not only in aortic ECs but in subaortic patches [19]. Runxl appears to be required for formation of intra-aortic HC clusters from ECs [20]. Several reports suggest that extrinsic regulation of AGM HSCs is also important. Thrombopoietin (TPO)/Mpl (TPO receptor) signaling plays a role in transition from hemogenic ECs to hematopoietic progenitor cells (HPCs)/HSCs via Runx1 activation and in production of HPCs/HSCs via Meis1 and Homeobox (Hox) b4 activation from $10.0 \mathrm{dpc}$ to $10.5 \mathrm{dpc}$ in AGM region [21]. Intereukin-1 (IL-1) reportedly functions as an important homeostatic regulator at 

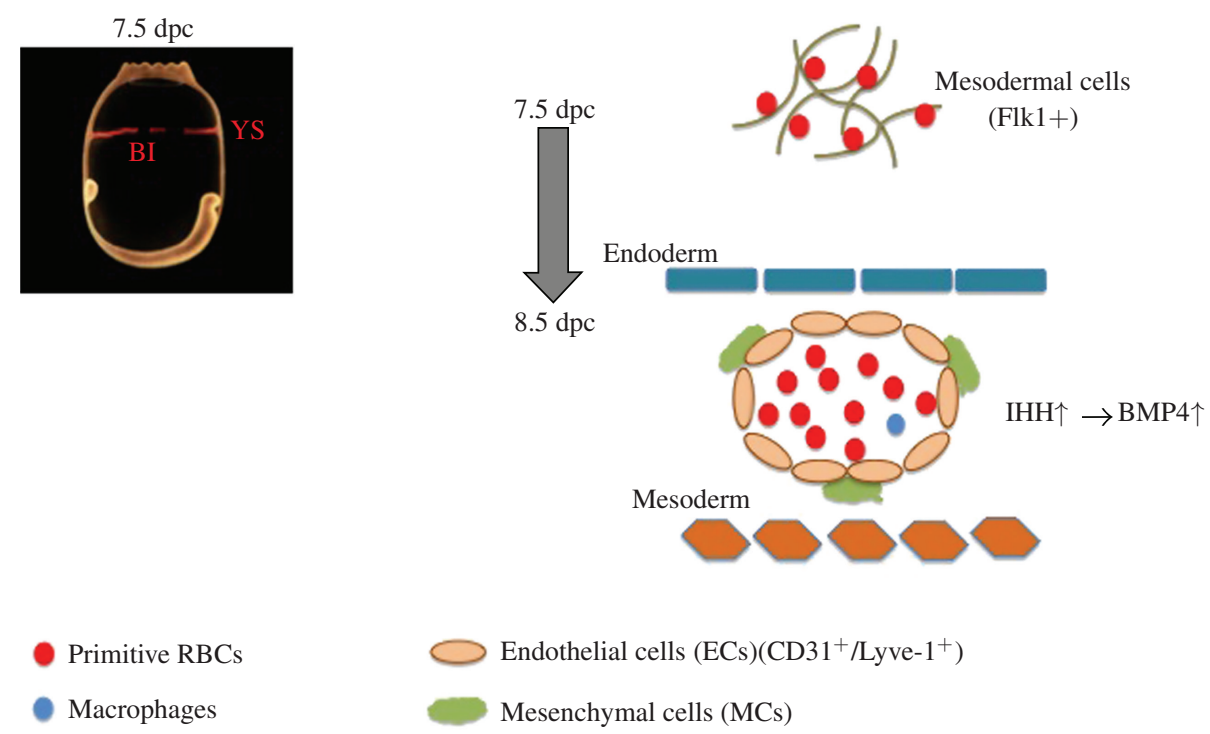

FIGURE 2: Hematopoietic environment in the YS. Mesodermal cells, the ancestors of hematopoietic cells, differentiate into both hematopoietic cells within blood islands and endothelial cells (ECs) outside of that region. Blood vessels, consisting of endothelial cells and containing primitive erythrocytes and macrophages, are located among cells originating from endodermal and mesodermal cells.

earliest stages of HSC development, limiting differentiation of some HSCs along the myeloid lineage [22] (Figure 3(b)). However, it remains unclear which factors regulate AGM HSCs. Since the AGM region consists of ECs, gonad cells, mesonephros cells, and unclassified MCs, those cells likely comprise a niche for HSC generation. c-Kit ${ }^{-} / \mathrm{CD} 34^{-} \mathrm{MCs}$ of the AGM region reportedly express hedgehog receptor/transducer genes, namely, Ptch1, Ptch2, and Smo, as well as genes encoding their downstream effectors, Gli1, Gli2, and Gli3, which act to increase the number of HSCs at $10.5 \mathrm{dpc}$. In addition, ECs $\left(\mathrm{CD} 31^{+} / \mathrm{CD} 34^{+} / \mathrm{Kit}^{-}\right)$of the AGM region at $10.5 \mathrm{dpc}$ likely function in $\mathrm{HSC}$ regulation through stem cell factor (SCF) secretion [23]. Taken together, the niche is comprised of several factors governing HSC generation.

\section{THE PLACENTA}

The PL is a highly vascularized structure that functions not only in gas exchange and fetal nutrition but in hematopoiesis at 8.5-13.5 dpc [24]. Similar to the AGM region, c-Kit-positive HC clusters in the PL are observed in the vitelline and umbilical arteries of the placenta (Figure 4(a)). A significant proportion of HPCs, including highly proliferative potential colony forming cells (HPP-CFCs), are detected in the mouse placenta [25]. HSCs (c-Kit $\left.{ }^{+} / \mathrm{CD} 34^{+}\right)$by $11.5 \mathrm{dpc}$, and the number increase until $12.5 \mathrm{dpc}$, resulting in a 15 fold increase in HSC activity compared with that of the AGM region [26, 27]. These findings indicate that mouse PL is a likely site for HSC generation and expansion at mid-gestation. Formation of HC clusters in both the AGM region and PL is impaired in mutant mice deficient in genes encoding specific transcription factors, namely, Runx1, Evi-1, and c-Myb, suggesting the importance of intrinsic regulation [7, 20, 28, 29]. Relevant to extrinsic regulation of PL HSCs, $I L-3$ not only is a target of Runx1 in adult HCs, but also regulates HSC proliferation in the PL [30]. We previously demonstrated regulation of HC clusters in the PL by surrounding niche cells [23]. To do so, we used laser-capture microdissection and flow cytometry to capture niche cells. Among several cytokine genes, only $S C F$ was expressed in niche cells, particularly in ECs. SCF protein expression was confirmed by ELISA and immunohistochemistry. Administration of blocking antibodies to c-Kit, the SCF receptor, clearly demonstrated that SCF/c-Kit signaling is pivotal for 


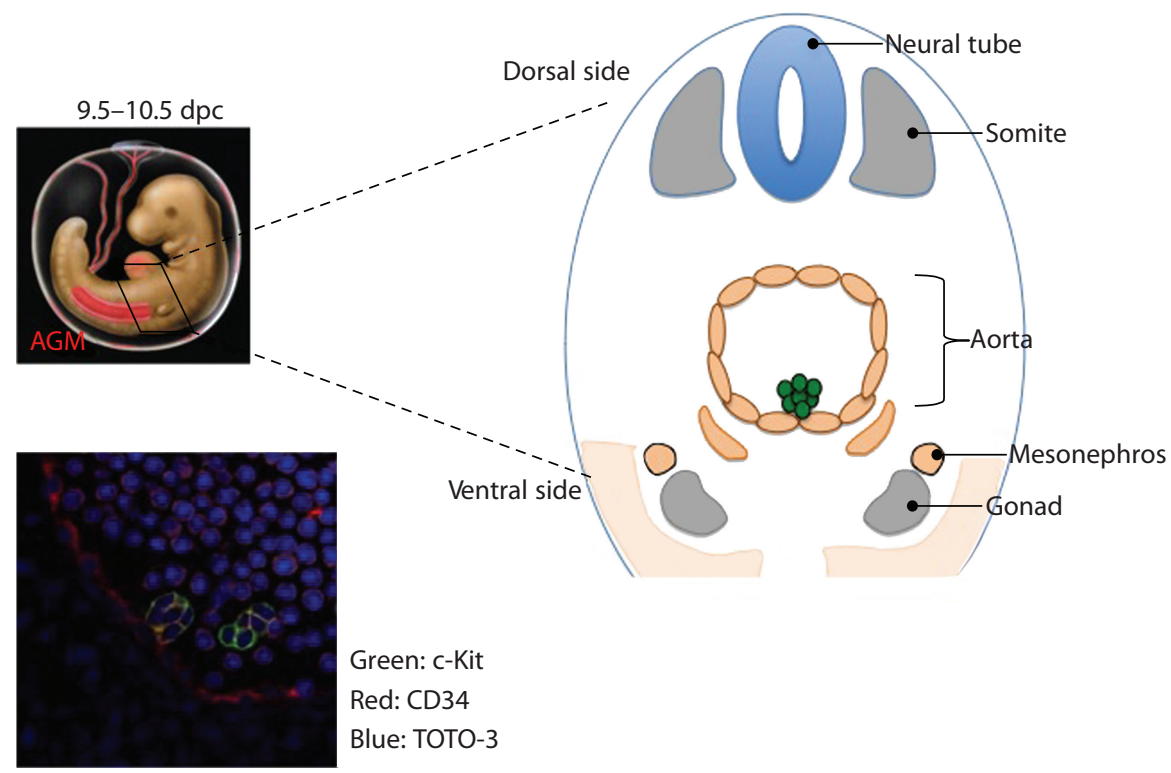

(a)

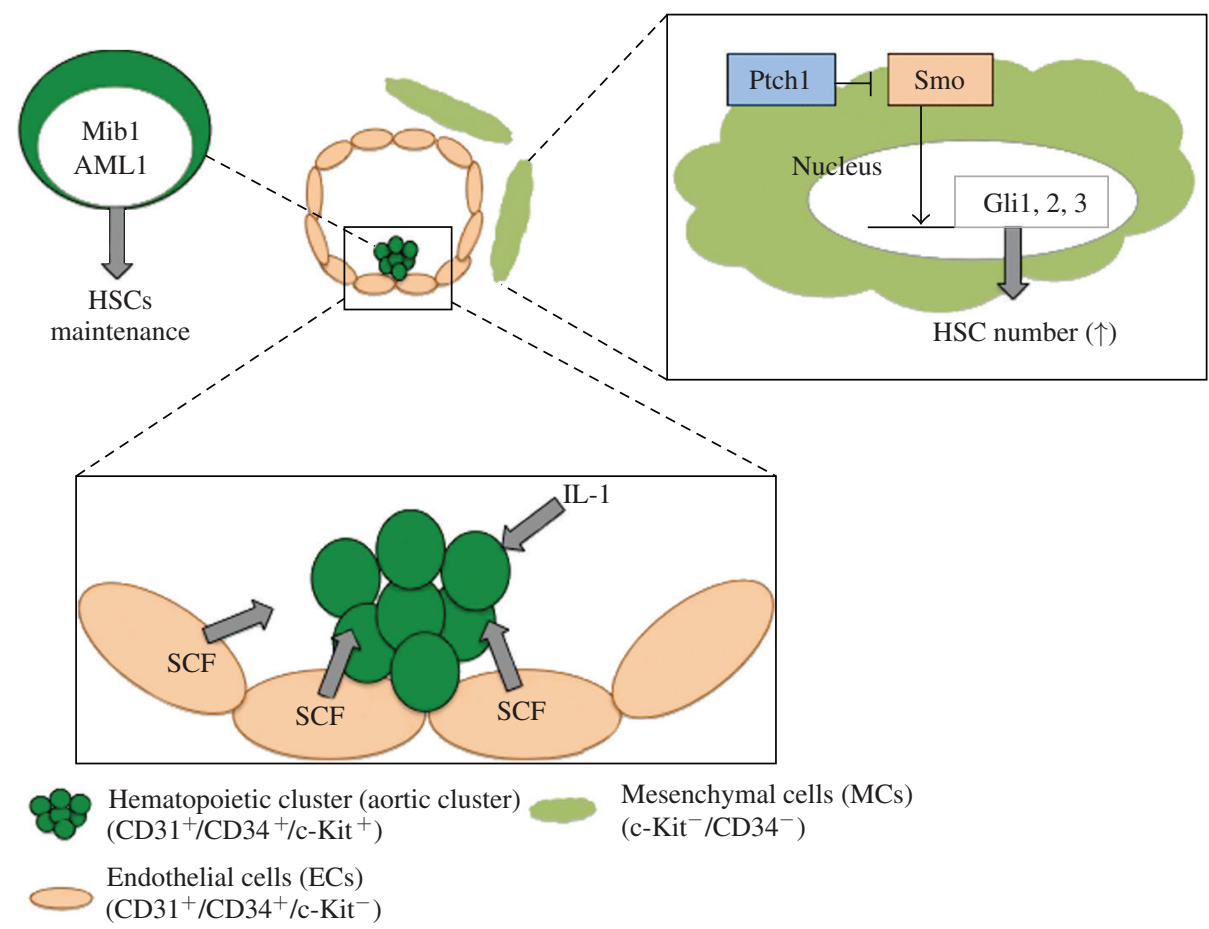

(b)

FIGURE 3: Hematopoietic environment in the AGM region. (a) (upper) Model of HC clusters, which are attached to the endothelial wall mainly on the ventral side. (lower) Confocal images of an HC cluster expressing CD34/c-Kit in the aortic region at $10.5 \mathrm{dpc}$. AGM region tissue sections were prepared from ICR mouse embryos at $10.5 \mathrm{dpc}$, stained with antibodies, and observed under confocal microscopy. CD34 (red), c-Kit (green), and TOTO-3 (blue). (b) Model of intrinsic and extrinsic regulation at the AGM region. (upper) Transcription factors (such as Runx1) maintain HSC development as intrinsic regulators. Signal transducers (such as Ptch and Smo) and transcription factor (such as Gli1, 2, 3) in mesenchymal cells maintain HSC number and development. (lower) Cytokines (SCF and IL-1) maintain HC clusters and HSC development as extrinsic regulators. 


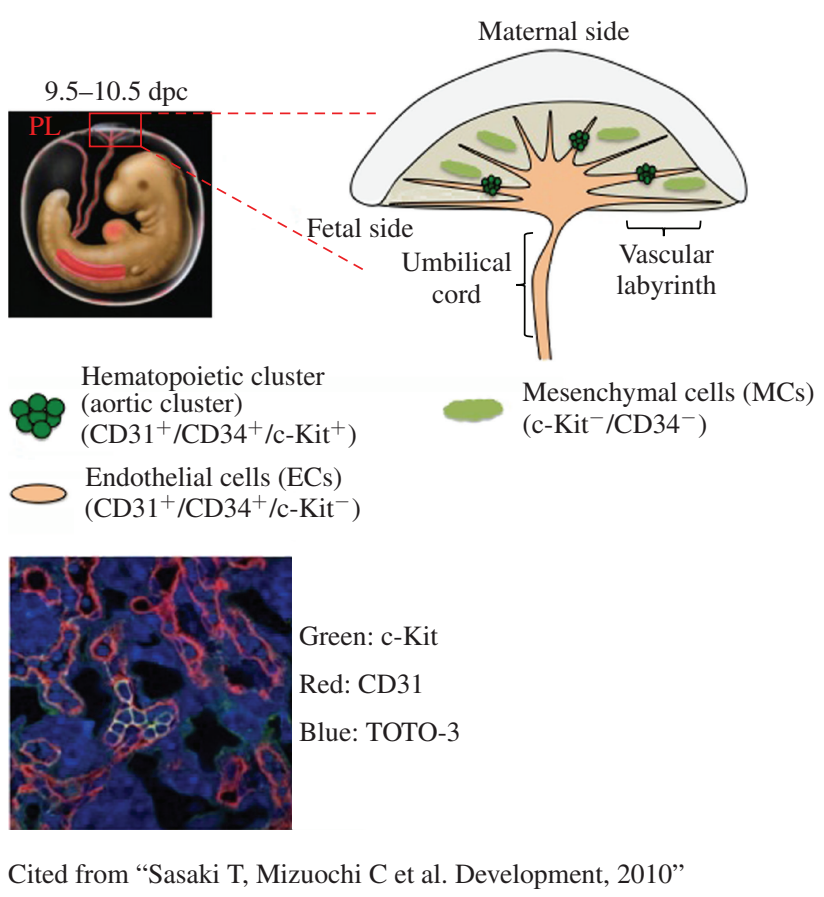

(a)

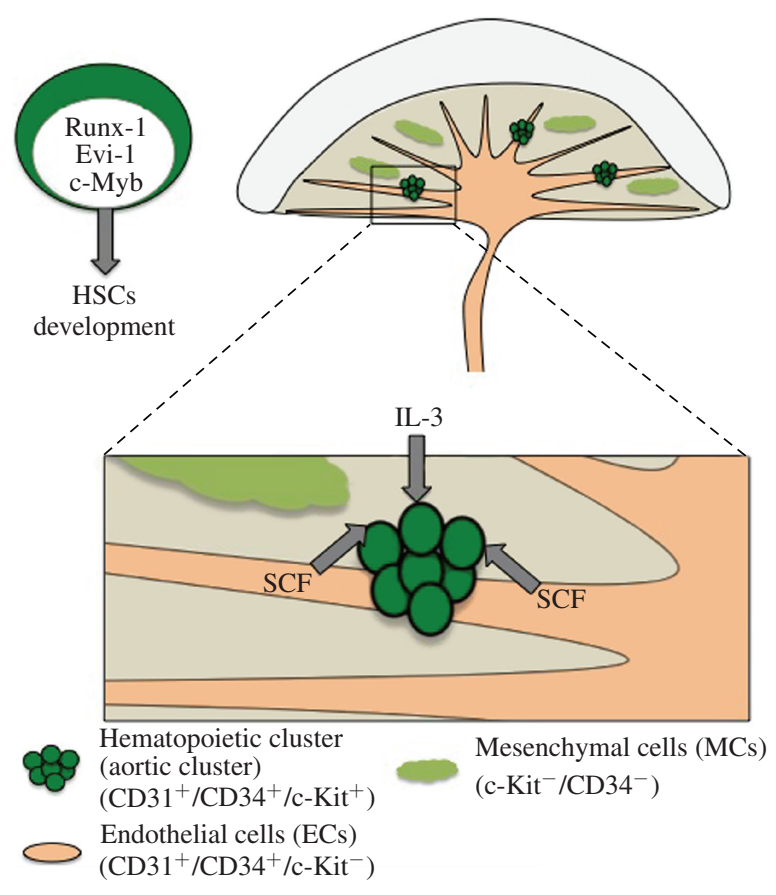

(b)

FIGURE 4: Hematopoietic environment in the placenta. (a) (upper) Model of HC clusters in the placenta. $\mathrm{HC}$ clusters are attached to the endothelial wall of capillary vessels, the so-called vascular labyrinth region, from $10.5 \mathrm{dpc}$ to $12.5 \mathrm{dpc}$. (lower) Confocal images of an HC cluster expressing CD31/c-Kit in the placenta at $10.5 \mathrm{dpc}$. Sections of placenta were prepared from ICR mouse embryos at $10.5 \mathrm{dpc}$, stained with indicated antibodies and observed under confocal microscopy. CD31 (red), Kit (green), and TOTO-3 (blue). (b) Model of intrinsic and extrinsic regulation in the placenta. (upper) Transcription factors (such as Runx1, Evi-1, cMyb) maintain HSC development intrinsically. (lower) Cytokines (such as SCF and IL-3) maintain HSCs as extrinsic regulators.

HSC regulation in the PL [23] (Figure 4(b)). Based on gene expression analysis, niche cells do not express $I L-3$ [23], although future studies are required to clarify its function in vivo.

\section{FETAL LIVER}

Hematopoiesis occurs in fetal liver (FL) by mid-gestation [31-33]. After HSCs are generated in the YS, AGM region, and PL, they migrate into FL, where HSCs are maintained and the number of HSCs dramatically increases between 12 and $16 \mathrm{dpc}$, during which time differentiation of mature HCs, particularly erythrocytes, occurs [32-34]. Thus FL is an organ appropriate for investigation of HSC expansion and erythropoiesis in vitro. FL HSCs express the markers c-Kit, stem cell antigen-1 (Sca-1), and protein tyrosine phosphatase, receptor type, $\mathrm{C}$ (CD45, also known as common leukocyte antigen). Circulating HPCs and HSCs expressing c-Kit have been reported to colonize FL [35]. Both morphological observation and experiments in vitro suggest that FL itself does not produce HSCs or HPCs but may be colonized by HCs of extrinsic origin after $9.5 \mathrm{dpc}[32,33,36,37]$. Intrinsic regulation of hematopoiesis in FL has also been reported. Factors that function in that activity include PU.1, an ETS family transcription factor, which functions in maintenance (differentiation) or expansion of FL HSCs [38]. Class I Hoxb4 and Hoxb3 also positively regulate HSC and HPC proliferation by accelerating the cell cycle [39]. Cited2 (a CBP/p300interacting transactivator with a glutamic acid [E] and aspartic acid [D]-rich tail 2) maintains FL HSCs and 


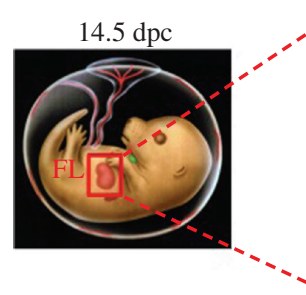

Hematopoietic cell $\left(\mathrm{CD}^{4} 5^{+} / \mathrm{Ter} 119^{+}\right)$ Sinusoid endothelial cells (Lyve- $1^{+} /$terCD $31^{+}$)

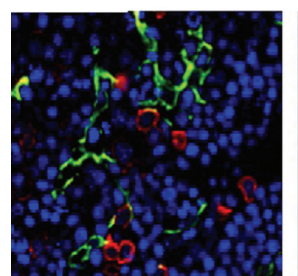

Green : Lyve-1 Red :c-Kit Blue :TOTO-3

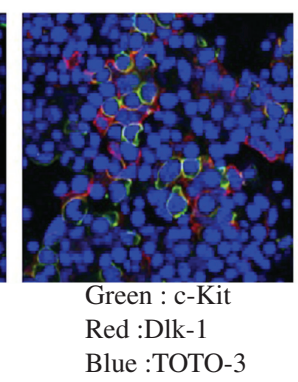

(a)
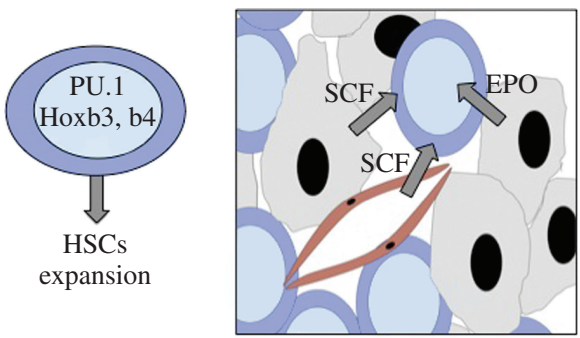

Hematopoietic cells $\left(\mathrm{CD}^{4} 5^{+} / \mathrm{Ter} 119^{+}\right)$ Sinusoid endothelial cells (Lyve- $1^{+} /$terCD31 ${ }^{+}$)

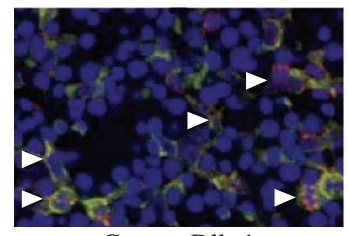

Green : Dlk-1

Red : EPO Blue : TOTO-3

Cited from "Sugiyama D, Kulkeaw K et al.

Biochemical and Biophysical Research Communications

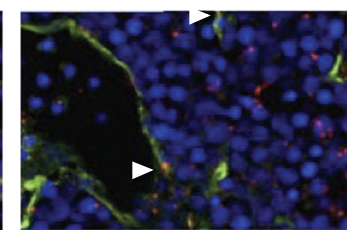

Green : Lyve-1 Red : SCF Blue : TOTO-3 (b)

FIGURE 5: Hematopoietic environment in fetal liver. (a) (upper) Structure of fetal liver, which primarily consists of hematopoietic cells, sinusoid endothelial cells, and hepatoblasts. (lower) Confocal images of FL expressing Dlk-1/c-Kit (Right). FL sections were prepared from ICR mouse embryos at $12.5 \mathrm{dpc}$, stained with indicated antibodies, and observed by confocal microscopy. c-Kit (green), Dlk-1 (red), and TOTO-3 (blue). (b) Intrinsic and extrinsic regulation in FL. (upper) Transcription factors (specifically, PU.1, Hoxb3, and Hoxb4) and cytokines (such as SCF and EPO) play important roles in HSC expansion and differentiation in FL. (lower) Liver sections were prepared from ICR mouse embryos at $12.5 \mathrm{dpc}$ and stained with Dlk-1 (green), EPO (red) and TOTO-3 (blue) (Left), and Dlk-1 (green), SCF (red), and TOTO-3 (blue) (Right).

HPCs [40]. Relevant to extrinsic regulation of FL hematopoiesis, FL consists of hepatoblasts (hepatocyte precursors), ECs, hematopoietic cells [41, 42], and other cells such as MCs (Figure 5(a)). Among them, we recently demonstrated that hepatoblasts expressing Dlk-1 function in HSC differentiation, particularly erythropoiesis, through secretion of erythropoietin (EPO), a cytokine regulating erythroid differentiation and SCF [43] (Figure 5(b)). In addition, hepatoblasts $\left(\mathrm{SCF}^{+} \mathrm{DLK}^{+}\right)$function at $15.5 \mathrm{dpc}$ in FL to increase the number of HSCs likely through production of several cytokines, angiopoietin-like 3 (Angptl3), insulinlike growth factor-2 (IGF2), SCF, and thrombopoietin (TPO) [44]. Taken together, hepatoblasts comprise a niche for HSC regulation through cytokine secretion. However, it remains unclear how HSCs maintain selfrenewal capacity under intense exposure to cytokines, which stimulate HSC differentiation. Also, in addition to cytokines, the extracellular matrix (ECM), including the interstitial matrix and basement membrane, interacts with cell surface receptors to regulate hematopoiesis extrinsically in FL. Integrins, which function as receptors for ECM, are important effectors of cell adhesion, differentiation, and migration. Particularly in FL hematopoiesis, beta-1 integrin (fibronectin receptor, CD29) is reportedly crucial for HSC colonization [45-48]. Interaction of integrin heterodimers with ECMs could function as a homing mechanism and enable HSCs and hematopoietic progenitor cells (HPCs) to reside in FL [49-51]. ECM proteins are produced in various cell types and interact with cytokines, enabling cells to interpret cytokine signaling in a particular context [52]. 


\section{FETAL SPLEEN AND BONE MARROW}

After the number of HSCs increases in the FL, those cells home to fetal-spleen, where HSCs differentiate at 13.5 to $14.5 \mathrm{dpc}$ [53]. Fetal spleen-derived stromal cells enable HSCs to differentiate primarily into macrophages $\left(\mathrm{F} 4 / 80^{+} \mathrm{Mac1}^{+}\right)$rather than lymphocytes. After transient hematopoiesis in fetal spleen, HSCs gradually home to BM after $16.5 \mathrm{dpc}$, where they remain during their entire lifetime. Although fetal BM is formed by $15.5 \mathrm{dpc}$, HSCs capable of self-renewal and which are positive for the signaling lymphocyte activation molecule (SLAM) family marker CD150 and negative for CD48 and CD41 cannot be detected in this tissue until $17.5 \mathrm{dpc}$, suggesting that other mechanisms govern the ability of HSCs to home to fetal BM during this period. Expression of genes encoding HSC migration factors, such as Cxcr4 (a chemokine receptor), Col4a1 (related to type IV collagen alpha1 synthesis), Mmp2, Timp2 (related to degradation and regulation of development), is significantly upregulated in HSCs during embryonic development [54].

\section{CONCLUDING REMARKS}

During embryogenesis, extrinsic regulation by niche cells is pivotal for HSC generation, expansion, differentiation, and maintenance. Increased knowledge of niche cell regulation should facilitate development of novel methods of in vitro generation, expansion, and differentiation for HSCs. Furthermore, if artificial niche components that replicate internal environment in vivo could maintain the status of stem cell, for example, cell cycle and quiescence, migration of both normal HSCs and leukemic stem cells might be directed as planned. The study of niche regulation of hematopoiesis should impact novel stem cell engineering, which could be applied for clinical therapies in the future.

\section{ABBREVIATIONS}

$\begin{array}{ll}\text { dpc: } & \text { Days post coitum } \\ \text { YS: } & \text { Yolk sac } \\ \text { BI: } & \text { Blood island } \\ \text { AGM: } & \text { Aorta-gonad-mesonephros } \\ \text { PL: } & \text { Placenta } \\ \text { FL: } & \text { Fetal liver } \\ \text { BM: } & \text { Bone marrow } \\ \text { HSC: } & \text { Hematopoietic stem cell } \\ \text { HPC: } & \text { Hematopoietic progenitor cell } \\ \text { HC: } & \text { Hematopoietic cell. }\end{array}$

\section{ACKNOWLEDGMENTS}

The authors acknowledge grant support from Health and Labour Sciences Research Grant from Ministry of Health, Labour and Welfare, and the Tokyo Biochemical Research Foundation. Dr. T. Inoue is supported by a research fellowship from the Japan Society for the Promotion of Science. Dr. K. Kulkeaw is supported by a research fellowship from the Tokyo Biochemical Research Foundation. The autors thank Dr. K. Akashi, Dr. K. Tani, Ms. S. Okayama and Dr. K. Srinoun for helpful discussion, and Dr. Elise Lamar for critical reading of the paper.

\section{REFERENCES}

[1] D. Sugiyama and K. Tsuji, "Definitive hematopoiesis from endothelial cells in the mouse embryo; a simple guide," Trends in Cardiovascular Medicine, vol. 16, no. 2, pp. 45-49, 2006. 
[2] S. T. Fraser and M. H. Baron, "Embryonic fates for extraembryonic lineages: new perspectives," Journal of Cellular Biochemistry, vol. 107, no. 4, pp. 586-591, 2009.

[3] M. Belaoussoff, S. M. Farrington, and M. H. Baron, "Hematopoietic induction and respecification of A-P identity by visceral endoderm signaling in the mouse embryo," Development, vol. 125, no. 24, pp. 5009-5018, 1998.

[4] M. A. Dyer, S. M. Farrington, D. Mohn, J. R. Munday, and M. H. Baron, "Indian hedgehog activates hematopoiesis and vasculogenesis and can respecify prospective neurectodermal cell fate in the mouse embryo," Development, vol. 128, no. 10, pp. 1717-1730, 2001.

[5] J. Palis, S. Robertson, M. Kennedy, C. Wall, and G. Keller, "Development of erythroid and myeloid progenitors in the yolk sac and embryo proper of the mouse," Development, vol. 126, no. 22, pp. 5073-5084, 1999.

[6] J. Palis, J. Malik, K. E. McGrath, and P. D. Kingsley, "Primitive erythropoiesis in the mammalian embryo," International Journal of Developmental Biology, vol. 54, no. 6-7, pp. 1011-1018, 2010.

[7] K. E. McGrath, P. D. Kingsley, A. D. Koniski, R. L. Porter, T. P. Bushnell, and J. Palis, "Enucleation of primitive erythroid cells generates a transient population of "pyrenocytes" in the mammalian fetus," Blood, vol. 111, no. 4, pp. 2409-2417, 2008.

[8] S. T. Fraser, J. Isern, and M. H. Baron, "Maturation and enucleation of primitive erythroblasts during mouse embryogenesis is accompanied by changes in cell-surface antigen expression," Blood, vol. 109, no. 1, pp. 343$352,2007$.

[9] J. Isern, Z. He, S. T. Fraser et al., "Single-lineage transcriptome analysis reveals key regulatory pathways in primitive erythroid progenitors in the mouse embryo," Blood, vol. 117, no. 18, pp. 4924-4934, 2011.

[10] D. M. Adelman, E. Maltepe, and M. C. Simon, "Multilineage embryonic hematopoiesis requires hypoxic ARNT activity," Genes and Development, vol. 13, no. 19, pp. 2478-2483, 1999.

[11] M. C. Yoder, K. Hiatt, P. Dutt, P. Mukherjee, D. M. Bodine, and D. Orlic, "Characterization of definitive lymphohematopoietic stem cells in the day 9 murine yolk sac," Immunity, vol. 7, no. 3, pp. 335-344, 1997.

[12] D. Sugiyama, M. Ogawa, K. Nakao et al., "B cell potential can be obtained from pre-circulatory yolk sac, but with low frequency," Developmental Biology, vol. 301, no. 1, pp. 53-61, 2007.

[13] C. T. Lux, M. Yoshimoto, K. McGrath, S. J. Conway, J. Palis, and M. C. Yoder, "All primitive and definitive hematopoietic progenitor cells emerging before E10 in the mouse embryo are products of the yolk sac," Blood, vol. 111, no. 7, pp. 3435-3438, 2008.

[14] K. E. Rhodes, C. Gekas, Y. Wang et al., "The emergence of hematopoietic stem cells is initiated in the placental vasculature in the absence of circulation," Cell Stem Cell, vol. 2, no. 3, pp. 252-263, 2008.

[15] M. C. Yoder, K. Hiatt, and P. Mukherjee, "In vivo repopulating hematopoietic stem cells are present in the murine yolk sac at day 9.0 postcoitus," Proceedings of the National Academy of Sciences of the United States of America, vol. 94, no. 13, pp. 6776-6780, 1997.

[16] S. T. Fraser, M. Ogawa, R. T. Yu, S. Nishikawa, M. C. Yoder, and S. I. Nishikawa, "Definitive hematopoietic commitment within the embryonic vascular endothelial-cadherin+ population," Experimental Hematology, vol. 30, no. 9, pp. 1070-1078, 2002.

[17] I. M. Samokhvalov, N. I. Samokhvalova, and S. I. Nishikawa, "Cell tracing shows the contribution of the yolk sac to adult haematopoiesis," Nature, vol. 446, no. 7139, pp. 1056-1061, 2007.

[18] K. Kumano, S. Chiba, A. Kunisato et al., "Notch1 but not Notch2 is essential for generating hematopoietic stem cells from endothelial cells," Immunity, vol. 18, no. 5, pp. 699-711, 2003.

[19] M. J. Yoon, B. K. Koo, R. Song et al., "Mind bomb-1 is essential for intraembryonic hematopoiesis in the aortic endothelium and the subaortic patches," Molecular and Cellular Biology, vol. 28, no. 15, pp. 4794-4804, 2008.

[20] T. North, T. L. Gu, T. Stacy et al., "Cbfa2 is required for the formation of intra-aortic hematopoietic clusters," Development, vol. 126, no. 11, pp. 2563-2575, 1999.

[21] M. Fleury, L. Petit-Cocault, D. Clay, and M. Souyri, "Mpl receptor defect leads to earlier appearance of hematopoietic cells/hematopoietic stem cells in the Aorta-Gonad-Mesonephros region, with increased apoptosis," International Journal of Developmental Biology, vol. 54, no. 6-7, pp. 1067-1074, 2010.

[22] C. Orelio, E. Haak, M. Peeters, and E. Dzierzak, "Interleukin-1 mediated hematopoietic cell regulation in the aorta-gonad-mesonephros region of the mouse embryo," Blood, vol. 112, no. 13, pp. 4895-4904, 2008.

[23] T. Sasaki, C. Mizuochi, Y. Horio, K. Nakao, K. Akashi, and D. Sugiyama, "Regulation of hematopoietic cell clusters in the placental niche through SCF/Kit signaling in embryonic mouse," Development, vol. 137, no. 23, pp. 3941-3952, 2010. 
[24] H. K. A. Mikkola, C. Gekas, S. H. Orkin, and F. Dieterlen-Lievre, "Placenta as a site for hematopoietic stem cell development," Experimental Hematology, vol. 33, no. 9, pp. 1048-1054, 2005.

[25] M. Alvarez-Silva, P. Belo-Diabangouaya, J. Salaün, and F. Dieterlen-Lièvre, "Mouse placenta is a major hematopoietic organ," Development, vol. 130, no. 22, pp. 5437-5444, 2003.

[26] C. Gekas, F. Dieterlen-Lièvre, S. H. Orkin, and H. K. A. Mikkola, "The placenta is a niche for hematopoietic stem cells," Developmental Cell, vol. 8, no. 3, pp. 365-375, 2005.

[27] K. Ottersbach and E. Dzierzak, "The murine placenta contains hematopoietic stem cells within the vascular labyrinth region," Developmental Cell, vol. 8, no. 3, pp. 377-387, 2005.

[28] T. Okuda, J. Van Deursen, S. W. Hiebert, G. Grosveld, and J. R. Downing, "AML1, the target of multiple chromosomal translocations in human leukemia, is essential for normal fetal liver hematopoiesis," Cell, vol. 84, no. 2, pp. 321-330, 1996.

[29] Q. Wang, T. Stacy, J. D. Miller et al., “ The CBFbeta subunit is essential for CBFalpha2 (AML1) function in vivo," Cell, vol. 87, pp. 697-708, 1996.

[30] C. Robin, K. Ottersbach, C. Durand et al., "An unexpected role for IL-3 in the embryonic development of hematopoietic stem cells," Developmental Cell, vol. 11, no. 2, pp. 171-180, 2006.

[31] A. L. Medvinsky and E. A. Dzierzak, "Development of the definitive hematopoietic hierarchy in the mouse," Developmental and Comparative Immunology, vol. 22, no. 3, pp. 289-301, 1998.

[32] G. R. Johnson and R. O. Jones, "Differentiation of the mammalian hepatic primordium in vitro. I. Morphogenesis and the onset of haematopoiesis," Journal of Embryology and Experimental Morphology, vol. 30, no. 1, pp. 8396, 1973.

[33] G. R. Johnson and M. A. S. Moore, "Role of stem cell migration in initiation of mouse foetal liver haemopoiesis," Nature, vol. 258, no. 5537, pp. 726-728, 1975.

[34] H. Ema and H. Nakauchi, "Expansion of hematopoietic stem cells in the developing liver of a mouse embryo," Blood, vol. 95, no. 7, pp. 2284-2288, 2000.

[35] D. Sugiyama, K. I. Arai, and K. Tsuji, "Definitive hematopoiesis from acetyl LDL incorporating endothelial cells in the mouse embryo," Stem Cells and Development, vol. 14, no. 6, pp. 687-696, 2005.

[36] E. Houssaint, "Differentiation of the mouse hepatic primordium. II. Extrinsic origin of the haemopoietic cell line," Cell Differentiation, vol. 10, no. 5, pp. 243-252, 1981.

[37] C. A. Cudenne and G. R. Johnson, "Presence of multipotential hemopoietic cells in teratocarcinoma cultures," Journal of Embryology and Experimental Morphology, vol. 61, pp. 51-59, 1981.

[38] H. G. Kim, C. G. De Guzman, C. Scott Swindle et al., "The ETS family transcription factor PU.1 is necessary for the maintenance of fetal liver hematopoietic stem cells," Blood, vol. 104, no. 13, pp. 3894-3900, 2004.

[39] J. M. Björnsson, N. Larsson, A. C. M. Brun et al., "Reduced proliferative capacity of hematopoietic stem cells deficient in Hoxb3 and Hoxb4," Molecular and Cellular Biology, vol. 23, no. 11, pp. 3872-3883, 2003.

[40] Y. Chen, P. Haviernik, K. D. Bunting, and Y. C. Yang, "Cited2 is required for normal hematopoiesis in the murine fetal liver," Blood, vol. 110, no. 8, pp. 2889-2898, 2007.

[41] C. M. Carreira, S. M. Nasser, E. Di Tomaso et al., "LYVE-1 is not restricted to the lymph vessels: expression in normal liver blood sinusoids and down-regulation in human liver cancer and cirrhosis," Cancer Research, vol. 61, no. 22, pp. 8079-8084, 2001.

[42] N. Tanimizu, M. Nishikawa, H. Saito, T. Tsujimura, and A. Miyajima, "Isolation of hepatoblasts based on the expression of Dlk/Pref-1," Journal of Cell Science, vol. 116, no. 9, pp. 1775-1786, 2003.

[43] D. Sugiyama, K. Kulkeaw, C. Mizuochi, Y. Horio, and S. Okayama, "Hepatoblasts comprise a niche for fetal liver erythropoiesis through cytokine production," Biochemical and Biophysical Research Communications, vol. 410, no. 2, pp. 301-306, 2011.

[44] S. Chou and H. F. Lodish, "Fetal liver hepatic progenitors are supportive stromal cells for hematopoietic stem cells," Proceedings of the National Academy of Sciences of the United States of America, vol. 107, no. 17, pp. 7799-7804, 2010.

[45] R. O. Hynes and K. M. Yamada, "Fibronectins: multifunctional modular glycoproteins," Journal of Cell Biology, vol. 95, no. 2 I, pp. 369-377, 1982.

[46] M. J. Humphries, M. Obara, K. Olden, and K. M. Yamada, "Role of fibronectin in adhesion, migration, and metastasis," Cancer Investigation, vol. 7, no. 4, pp. 373-393, 1989. 
[47] E. Hirsch, A. Iglesias, A. J. Potocnik, U. Hartmann, and R. Fässler, "Impaired migration but not differentiation of haematopoietic stem cells in the absence of $\beta 1$ integrins," Nature, vol. 380, no. 6570, pp. 171-175, 1996.

[48] S. M. Frisch and E. Ruoslahti, "Integrins and anoikis," Current Opinion in Cell Biology, vol. 9, no. 5, pp. 701706, 1997.

[49] V. P. Patel and H. F. Lodish, "A fibronectin matrix is required for differentiation of murine erythroleukemia cells into reticulocytes," Journal of Cell Biology, vol. 105, no. 6, pp. 3105-3118, 1987.

[50] M. W. Long and V. M. Dixit, "Thrombospondin functions as a cytoadhesion molecule for human hematopoietic progenitor cells," Blood, vol. 75, no. 12, pp. 2311-2318, 1990.

[51] E. S. Strobel, D. Möbest, S. Von Kleist et al., "Adhesion and migration are differentially regulated in hematopoietic progenitor cells by cytokines and extracellular matrix," Blood, vol. 90, no. 9, pp. 3524-3532, 1997.

[52] J. Taipale and J. Keski-Oja, "Growth factors in the extracellular matrix," The FASEB Journal, vol. 11, no. 1, pp. 51-59, 1997.

[53] J. Y. Bertrand, G. E. Desanti, R. Lo-Man, C. Leclerc, A. Cumano, and R. Golub, "Fetal spleen stroma drives macrophage commitment," Development, vol. 133, no. 18, pp. 3619-3628, 2006.

[54] J. Ciriza and M. E. García-Ojeda, "Expression of migration-related genes is progressively upregulated in murine Lineage-Sca-1+c-Kit+ population from the fetal to adult stages of development," Stem Cell Research and Therapy, vol. 1, no. 2, article 14, 2010.

\section{This article should be cited as follows:}

Daisuke Sugiyama, Tomoko Inoue-Yokoo, Stuart T. Fraser, Kasem Kulkeaw, Chiyo Mizuochi, and Yuka Horio, "Embryonic Regulation of the Mouse Hematopoietic Niche," TheScientific WorldJOURNAL, vol. 11, pp. 1770-1780, 2011. 

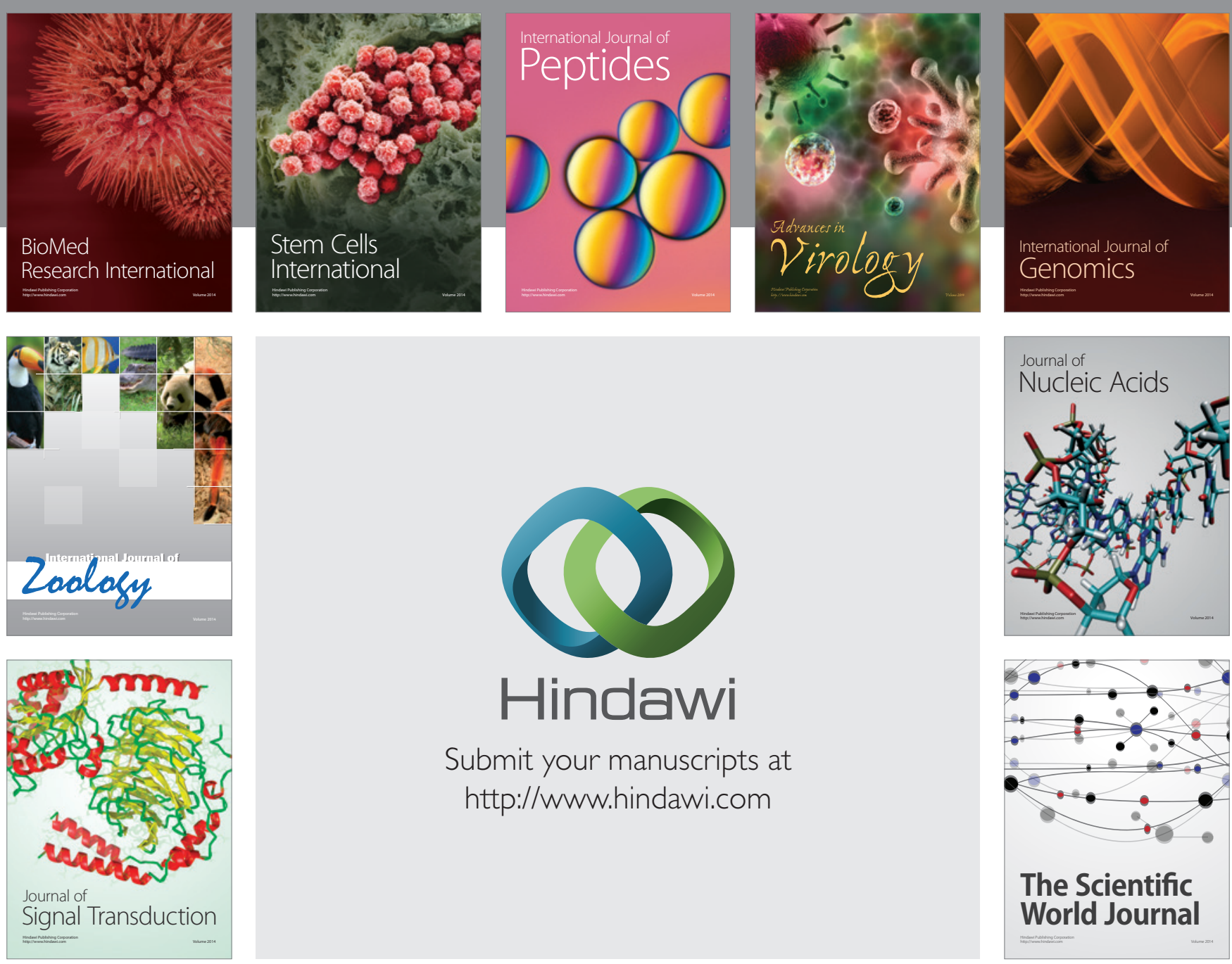

Submit your manuscripts at

http://www.hindawi.com
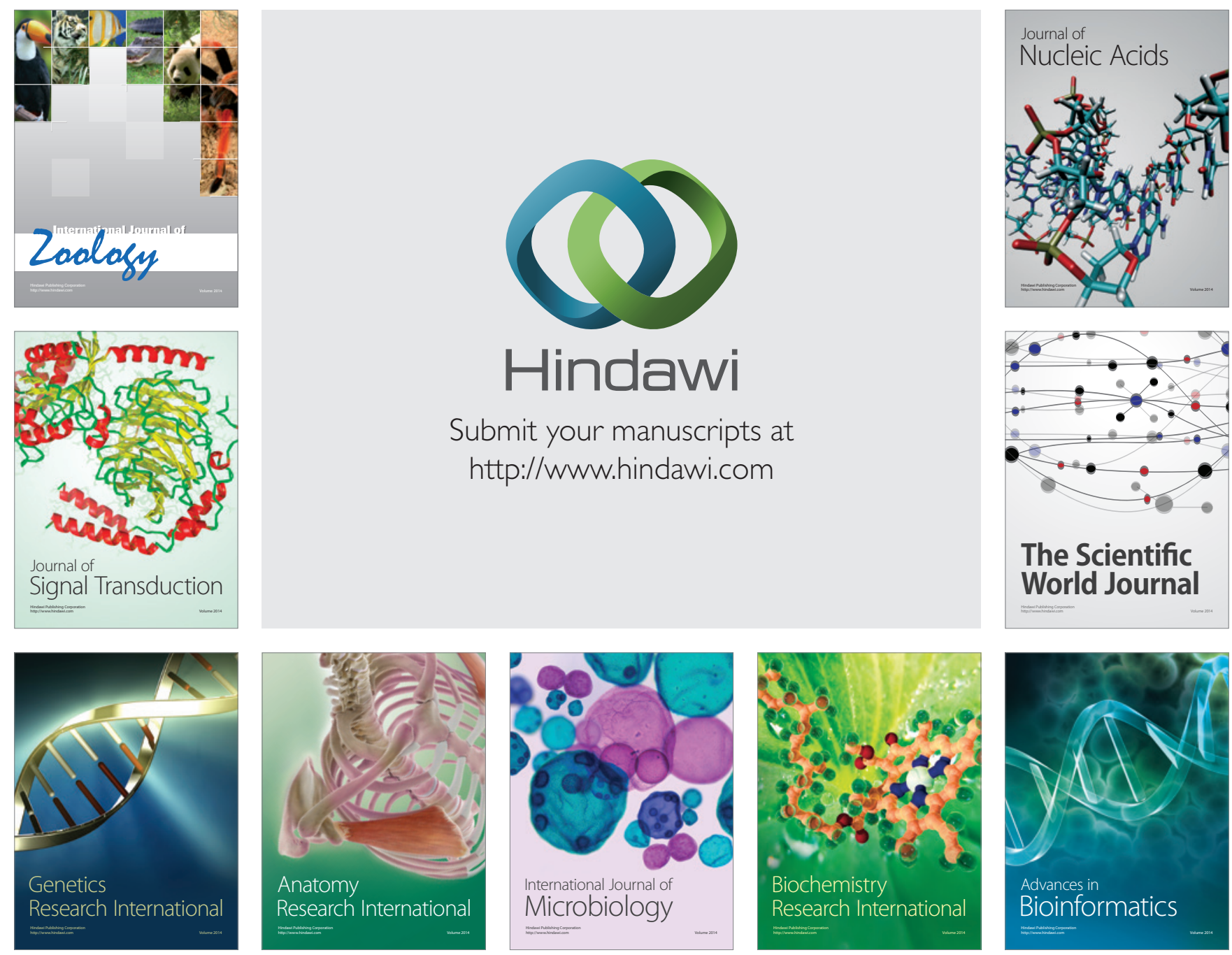

The Scientific World Journal
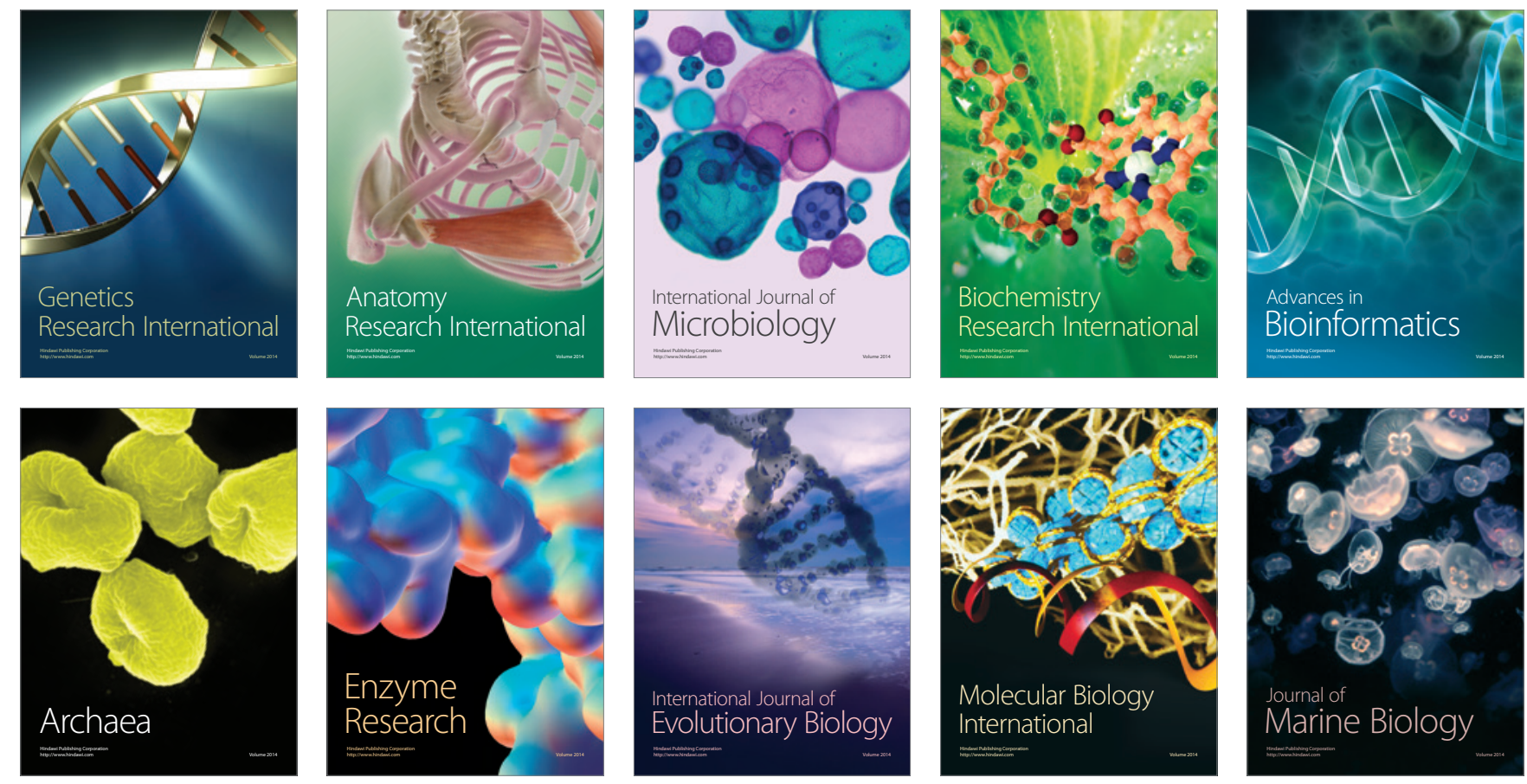